\title{
COALIZÃO TARSAL CUBOIDE-NAVICULAR
}

\section{CUBOID-NAVICULAR TARSAL COALITION}

\author{
Marcelo Pires Prado', Alberto Abussamara Moreira Mendes², Rogério Olivi' ${ }^{1}$, Daniel Tassetto Amodio²
}

\section{RESUMO}

Os autores apresentam caso de paciente do sexo feminino de nove anos de idade que apresentava dores no pé direito associadas à prática de atividades físicas. Após o diagnóstico de coalizão tarsal cuboide-navicular foi tratada cirurgicamente através de ressecção desta, com resolução dos sintomas. Realizam revisão da literatura e discutem a importância da adequada avaliação clínica e imagenológica dos casos das dores no pé nas crianças e adolescentes.

Descritores - Deformidades Congênitas do Pé/cirurgia; Ossos do Tarso/cirurgia; Articulação Talocalcânea

\section{ABSTRACT}

The authors present a case of a 9-year-old female patient who presents with complaints of pain in her right foot associated with physical activities. After the diagnosis of an osseous cuboid navicular coalition she was treated surgically with resection of the coalition, with symptoms resolved. The literature is reviewed and the importance of adequate physical examination and image exams to investigate foot pain in children and teenagers is discussed.

Keywords - Foot Deformities, Congenital/surgery; Tarsal Bones/surgery; Subtalar Joint

\section{INTRODUÇÃO}

A coalizão tarsal é uma anormalidade congênita em que ocorrem graus variáveis de fusão entre dois ou mais ossos do tarso. Acomete aproximadamente $2 \%$ da população e muitas vezes é assintomática ${ }^{(1)}$. A importância clínica desta patologia foi proposta por Holl, em 1880, que descreveu a relação entre as barras intertarsais e o pé plano rígido e doloroso ${ }^{(2)}$. Pode, eventualmente, apresentar-se como pé cavo varo rígido por espasmo do músculo tibial anterior ${ }^{(3)}$. As coalizões podem ser ósseas, cartilaginosas ou fibrosas. Os locais mais comuns das pontes ósseas são entre o tálus e o calcâneo (talocalcaneana medial) e entre o navicular e o calcâneo, correspondendo a $90 \%$ destas, respectivamente com $48 \%$ e $44 \%$ dos casos de coalizão tarsal. As barras entre o tálus e o navicular e entre o calcâneo e o cuboide correspondem a adicionais $1 \%$ cada. Johnson et al ${ }^{(4)}$ relatam caso de barra cubonavicular tratada cirurgicamente através de ressecção. A barra entre o cuboide e o navicular é rara, com poucos casos descritos na literatura ${ }^{(5-10)}$.

\section{RELATO DE CASO}

Criança de nove anos, sexo feminino, com queixa de desconforto no pé direito relacionado à prática de exercícios físicos há um ano, já havia sido tratada como tendinite e entorse do tornozelo, sem melhora do quadro. O tratamento incluiu restrição da atividade habitual, períodos de imobilização, fisioterapia, uso de palmilhas e medicamentos anti-inflamatórios não hormonais.

Clinicamente, apresentava-se com marcha normal, pé plantígrado, com diminuição da varização ativa do retropé quando ficava na ponta dos pés. Havia limitação da mobilidade da mediotársica, com mobilidade do tornozelo e da articulação subtalar preservadas.

A paciente foi encaminhada para tomografia computadorizada que evidenciou a presença de barra óssea entre o cuboide e o navicular (Figuras 1, 2, 3 e 4).

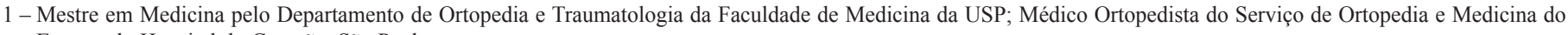
Esporte do Hospital do Coração, São Paulo.

2 - Médico Ortopedista do Serviço de Ortopedia e Medicina do Esporte do Hospital do Coração, São Paulo. 


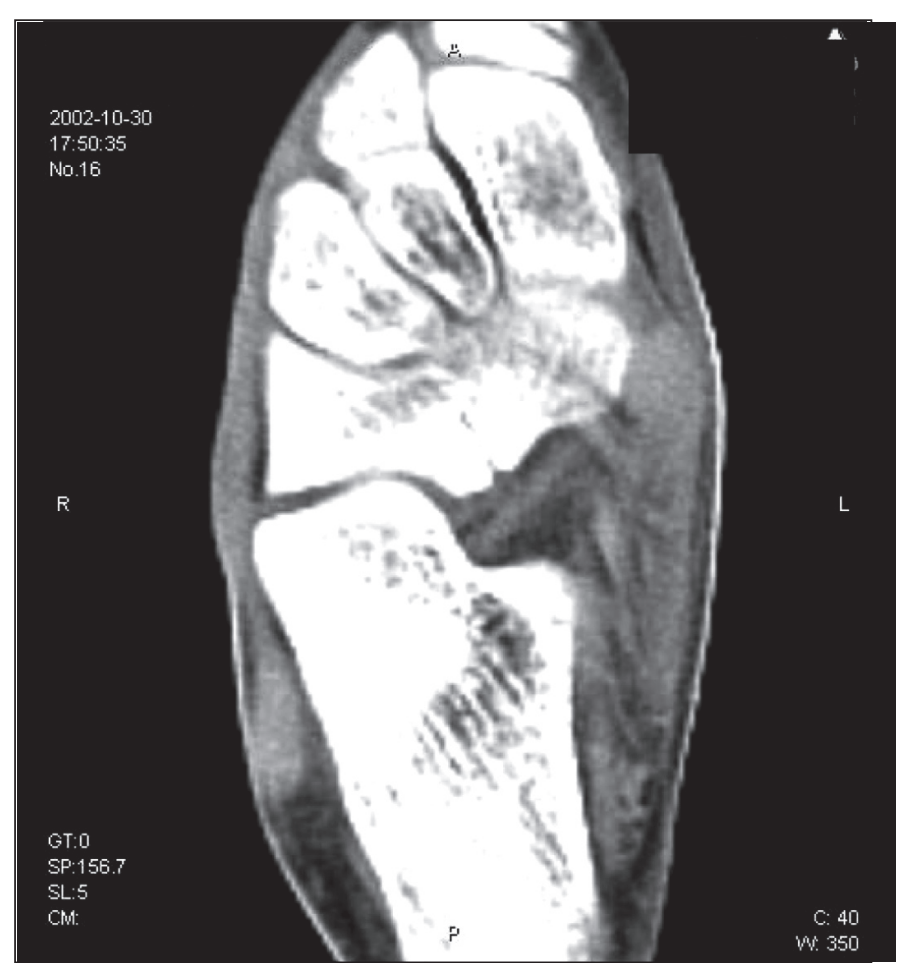

Figura 1 - Corte tomográfico mostrando a coalizão cuboidenavicular.

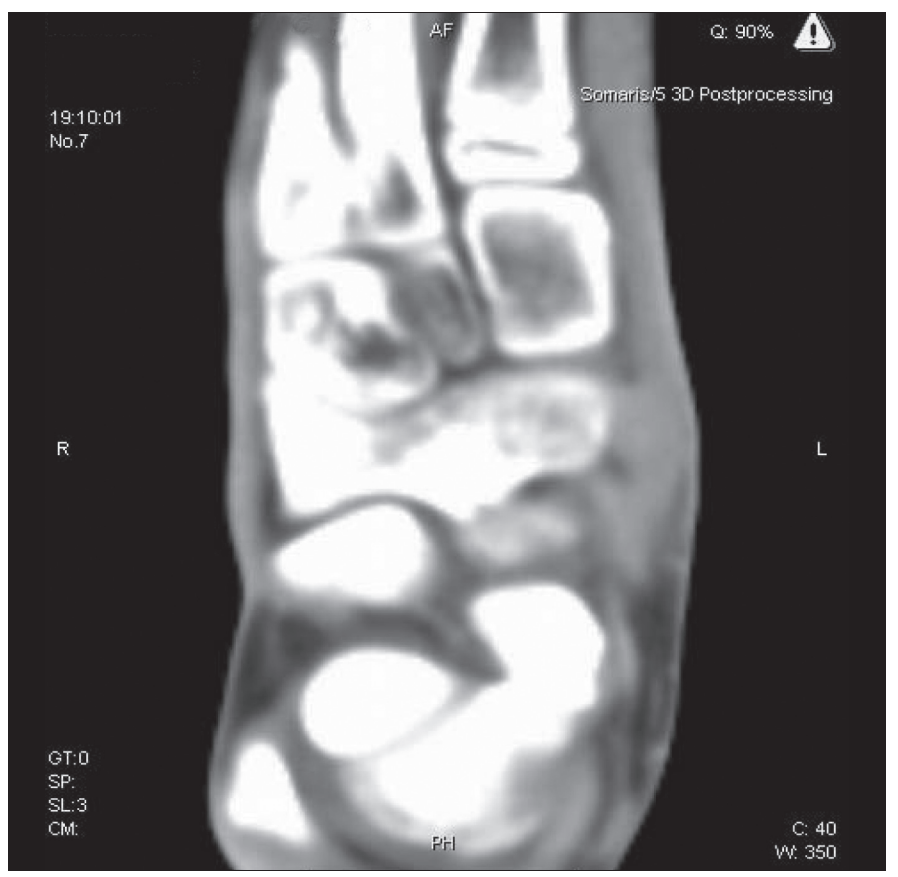

Figura 2 - Reconstrução tomográfica mostrando coalizão cuboide-navicular.

Nesta paciente, optamos pelo tratamento cirúrgico porque havia queixa contínua por pelo menos um ano, sem melhora com o tratamento conservador instituído, e já havia limitação da mobilidade da mediotársica.

Foi realizada incisão oblíqua na face lateral do pé (via de Ollier), com desinserção da origem do músculo

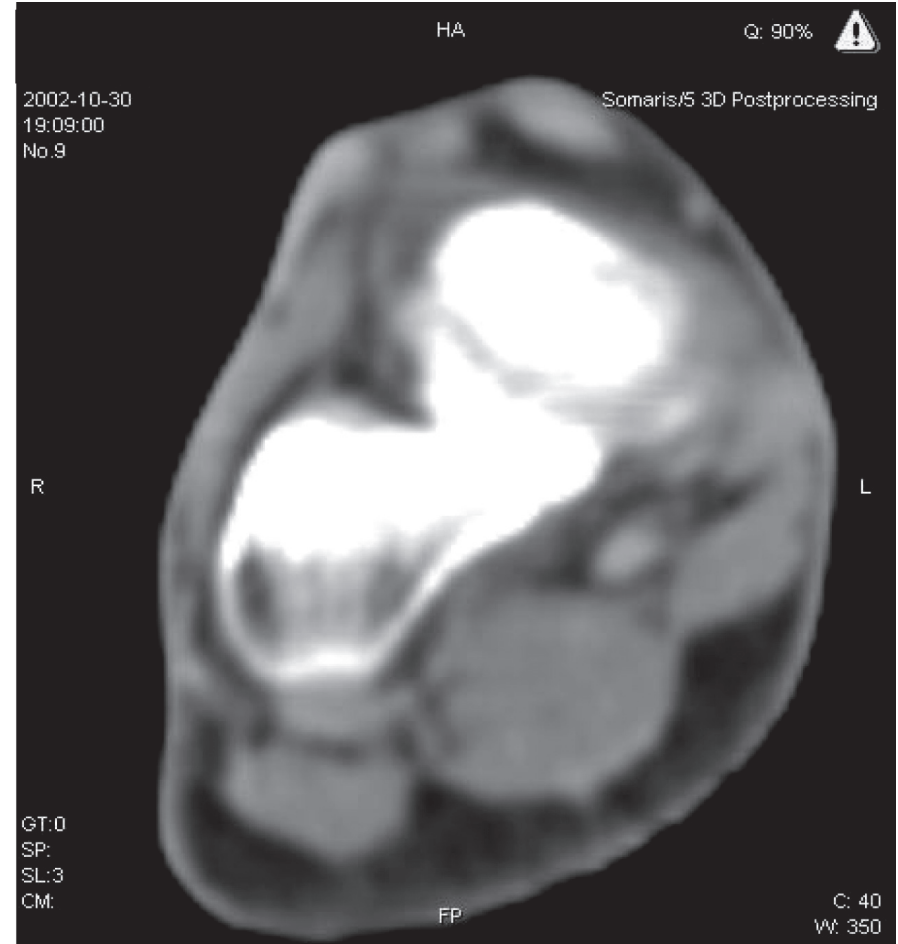

Figura 3 - Reconstrução tomográfica mostrando coalizão cuboide-navicular.

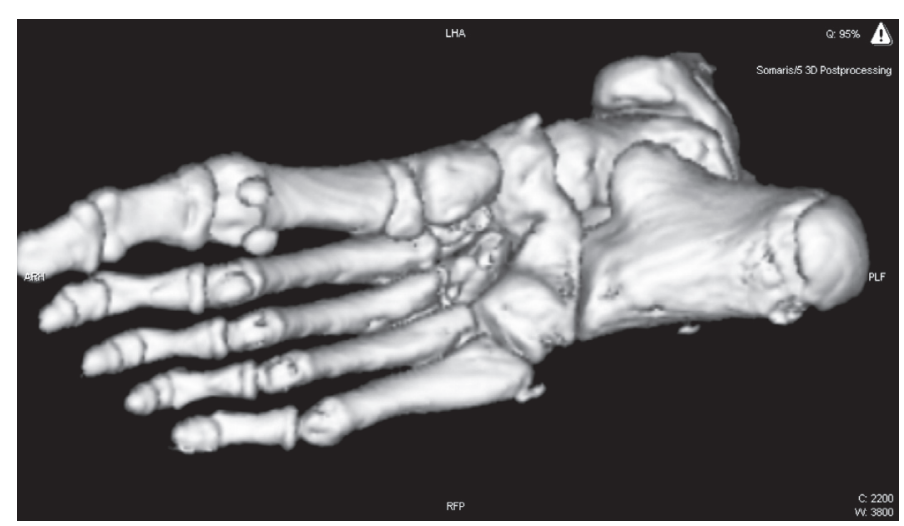

Figura 4 - Reconstrução tomográfica tridimensional mostrando coalizão cuboide-navicular.

extensor curto dos dedos, que é afastado para distal, o que permite a visualização da porção distal do calcâneo, cabeça do tálus, cuboide e navicular. Foi realizada a ressecção da barra entre o cuboide e o navicular e posterior interposição do músculo extensor curto dos dedos, à semelhança da técnica de Cowell para ressecção da barra calcaneonavicular ${ }^{(11,12)}$.

No pós-operatório, a paciente foi mantida sem apoio, andando com o auxílio de muletas por três semanas, com manipulação passiva e ativa da articulação subtalar e mediotársica desde o segundo dia. Em seguida, foi liberada para o apoio sem auxílio e intensificada a fisioterapia, no sentido do retorno da força muscular, propriocepção e treino de marcha. Após oito 
semanas do procedimento, a paciente estava apta a retornar às suas atividades prévias, e, após o terceiro mês de pós-operatório, assintomática, independente da atividade realizada.

Com cinco anos de pós-operatório, a paciente continua sem queixas, com o exame físico normal e sem limitações para as atividades físicas.

\section{DISCUSSÃO}

Segundo Tachdjian, muitos pacientes com coalizão tarsal não têm sintomas significantes e por isso não necessitam de tratamento. Na presença de sintomas agudos após trauma, o tratamento conservador pode ser indicado, com repouso, analgesia e observação da evolução. O tratamento conservador das coalizões inclui elevação do calcâneo, uso de órteses, e até imobilizações gessadas ${ }^{(5,13)}$. O tratamento cirúrgico está indicado quando ocorre falha no controle da dor com o tratamento conservador. A ressecção da barra é indicada nos pacien- tes jovens sem evidências radiográficas de alterações degenerativas ou associações com outras coalizões. A ressecção da coalizão proporciona bons resultados com o que concordam a maioria dos autores ${ }^{(14)}$. A via de acesso dorso lateral utilizada nesta paciente permite adequada visualização da barra e adequada ressecção evitando as complicações relacionadas às incisões plantares ${ }^{(4,15)}$. Nos pacientes com degeneração articular, coalizões múltiplas e falhas na ressecção deve-se considerar a indicação das artrodeses ${ }^{(16)}$.

A importância deste caso apresentado está no fato de que existem causas de pé plano valgo na infância que necessitam de tratamento específico. Nos pacientes com pé plano valgo sintomático, o médico deve suspeitar e investigar a presença de afecções associadas. Quando se observa rigidez articular no retro ou mediopé, as coalizões são a principal causa ${ }^{(16)}$, e a barra cuboide-navicular tem interesse pela raridade com que ocorre, principalmente associada a sintomas; no entanto, deve ser incluída no rol de possibilidades diagnósticas nestas circunstâncias.

\section{REFERÊNCIAS}

1. Stormont DM, Peterson HA. The relative incidence of tarsal coalition. Clin Orthop Relat Res. 1983;(181):28-36

2. Holl M. Beitrage zur chirurgischen osteologie dês fusses. Arch Klin Chir. 1880;25:211.

3. Tachdjian MO. The foot and leg. Tarsal coalition.In: Tachdjian MO. Pediatric orthopedics. 2a. ed. Philadelphia: Saunders; 1990. p. 2578-611.

4. Johnson TR, Mizel MS, Temple T. Cuboid-navicular tarsal coalition -- presentation and treatment: a case report and review of the literature. Foot Ankle Int. 2005;26(3):264-6.

5. Waugh W. Partial cubo-navicular coalition as a cause of peroneal spastic flat foot. J Bone Joint Surg Br. 1957;39(3):520-3.

6. Del Sel JM, Grand NE. Cubo-navicular synostosis; a rare tarsal anomaly. J Bone Joint Surg Br. 1959;41(1):149.

7. Cavallaro DC, Hadden HR. An unusual case of tarsal coalition: a cuboid navicular synostosis. J Am Podiatry Assoc. 1978;68(2):71-5.

8. Palladino SJ, Schiller L, Johnson JD. Cubonavicular coalition. J Am Podiatr Med Assoc. 1991;81(5):262-6.
9. Talkhani IS, Laing P. Cuboid-navicular coalition in an adult: a case report. Foot Ankle. 1999;5:151-4

10. Piqueres X, de Zabala S, Torrens C, Marin M. Cubonavicular coalition: a case report and literature review. Clin Orthop Relat Res. 2002;(396):112-4

11. Cowell HR, Elener V. Rigid painful flatfoot secondary to tarsal coalition. Clin Orthop Relat Res. 1983;(177):54-60.

12. Cowell HR. Extensor brevis arthroplasty. In: Proceedings of $37^{\text {th }}$ Annual Meeting of the American Academy of Orthopedic Surgeons. J Bone Joint Surg Am. 1970;52:820.

13. Kulik SA Jr, Clanton TO. Tarsal coalition. Foot Ankle Int. 1996;17(5):286-96

14. Richardson EG, Brotzman SB, Graves SC. The plantar incision for procedures involving the forefoot. An evaluation of one hundred and fifty incisions in one hundred and fifteen patients. J Bone Joint Surg Am. 1993;75(5):726-31.

15. Vincent KA. Tarsal coalition and painful flatfoot. J Am Acad Orthop Surg. 1998;6(5):274-81.

16. Harris RI. Retrospect--personeal spastic flat foot (rigid valgus foot). J Bone Joint Surg Am. 1965;47(8):1657-67. 